\title{
BMJ Open Impact of depression and/or anxiety on patients with percutaneous coronary interventions after acute coronary syndrome: a protocol for a real-world prospective cohort study
}

\author{
Yihui Xiao, ${ }^{1}$ Wenyuan Li, ${ }^{1}$ Juan Zhou, ${ }^{2}$ Jie Zheng, ${ }^{3}$ Xiaojie Cai, ${ }^{1}$ Manyun Guo, ${ }^{1}$ \\ Xiang Hao, ${ }^{1}$ Zhanyi Zhang, ${ }^{1}$ Yan Liu, ${ }^{1}$ Zuyi Yuan ${ }^{1,4}$
}

To cite: Xiao Y, Li W, Zhou J, et al. Impact of depression and/or anxiety on patients with percutaneous coronary interventions after acute coronary syndrome: a protocol for a real-world prospective cohort study. BMJ Open 2019;9:e027964. doi:10.1136/ bmjopen-2018-027964

\section{- Prepublication history for} this paper is available online. To view these files please visit the journal online (http://dx.doi. org/10.1136/bmjopen-2018027964).

YX and WL are joint first authors.

Received 16 November 2018

Revised 26 July 2019

Accepted 31 July 2019
Check for updates

(C) Author(s) (or their employer(s)) 2019. Re-use permitted under CC BY-NC. No commercial re-use. See rights and permissions. Published by BMJ.

For numbered affiliations see end of article.

Correspondence to

Dr Zuyi Yuan;

zuyiyuan@mail.xjtu.edu.cn

\section{ABSTRACT}

Introduction Acute coronary syndrome (ACS) is one of the leading causes of death. Depression and/or anxiety after ACS is common. Studies from developed countries have reported that the occurrence of anxiety or depression after ACS might increase the risk of cardiovascular events and mortality. However, the results varied, and are limited in developing countries. Therefore, well designed large-scale real-world study is needed to make further clarification. The main objective of this study is to evaluate whether depression or anxiety could affect the prognosis of patients with percutaneous coronary intervention (PCI) post-ACS.

Method and analysis The study is a prospective, multicentre, cohort study, which will be performed at 12 large hospitals in northwest China and led by the First Affiliated Hospital of Xi'an Jiaotong University. A total of 5000 patients with $\mathrm{PCI}$ post-ACS will be enrolled and followed up for 2 years. Their depression and anxiety status will be evaluated with the Patient Health Questionnaire- 9 or Generalised Anxiety Disorder-7 Assessment scales during the follow-up. A Cox proportional hazard model will be used to determine if depression/anxiety after $\mathrm{PCl}$ increase the risk of cardiovascular events. The impact of antidepression or antianxiety treatment on the cardiac prognosis will be explored as well among the patients with ACS who received the treatment after PCl.

Ethics and dissemination This study has been approved by the ethics committee of the First Affiliated Hospital of Xi'an Jiaotong University (approval number: XJTU1AF2016LSL-036). The results will be published in research articles or conference papers.

Trial registration number NCT03057691.

\section{INTRODUCTION}

Cardiovascular disease has been the leading cause of death in China, while acute coronary syndrome (ACS) accounts for $50 \%$ of cardiovascular deaths. ${ }^{1}$ ACS is the most common and high-risk type of coronary heart disease (CHD), including unstable angina (UA), non-ST-segment elevation myocardial infarction and ST-segment elevation myocardial infarction.

\section{Strengths and limitations of this study}

- This is the first multicentre real-world study in China, as well as developing countries focusing on post-acute coronary syndrome (ACS) depression and anxiety.

- To our knowledge, this is the largest project to evaluate the impact of depression and/or anxiety on the prognosis of patients with percutaneous coronary intervention (PCI) post-ACS, which will provide strong evidence for depression and/or anxiety being an independent risk factor for patients with $\mathrm{PCl}$ post-ACS.

- This is the largest study to provide real-world information about the occurrence and therapeutic acceptance of depression and/or anxiety.

- Some inadequacy of data recording and inter-centre selection bias may occur, which are limitations that are common to multicentre cohort studies.

Percutaneous coronary intervention (PCI) is the most important treatment for ACS at present. However, patients with PCI post-ACS still suffer a high incidence of major adverse cardiovascular events (MACE). ${ }^{2}$ Studies have shown that risk factors (such as hypertension, diabetes, and smoking) are associated with the prognosis after PCI. ${ }^{3-5}$ Recently, an increasing number of studies have indicated that anxiety and/or depression following CHD was very common, especially in ACS. ${ }^{67}$

The incidence of post-ACS anxiety in developed countries is extensive in that about $20 \%-60 \%$ of patients with acute myocardial infarction (MI) developed symptoms of anxiety. ${ }^{8} 9$ However, there are not enough data on the incidence of anxiety in post-ACS patients in China, and few studies have focused on the prognosis of patients with post-ACS anxiety throughout the world. 
Studies from developed countries have reported that the incidence of post-ACS depression is about 15\%-20\% within 1 year, which is three times as many as that of the general population. ${ }^{10}$ Moreover, the occurrence of depression after PCI has been found to be associated with adverse cardiac outcomes and mortality in patients with ACS. ${ }^{811}$ Therefore, in 2008, the American Heart Association (AHA) recommended routine screening of depression in patients with CHD to guide treatment options, ${ }^{12}$ and in 2014, the AHA proposed depression as a risk factor for adverse outcomes in patients with ACS. ${ }^{13}$ However, there are no large clinical studies showing the relationship between depression and ACS in China.

Although the incidence of anxiety and depression after ACS has been depicted in many studies in developed countries, conditions in developing countries like China have not been well elucidated. Meanwhile, unlike the detrimental impact of depression on the prognosis of patients with ACS, the impact of anxiety post-ACS remains ambiguous due to the lack of relevant research. Hence, the main objective of this study is to evaluate the cardiac effect of depression and/or anxiety on the prognosis of these patients with PCI post-ACS. Furthermore, if our study could prove that depression and/or anxiety is the important risk factor for adverse cardiac events, we will further explore whether antidepressant treatment or improvement of the emotional status could improve the prognosis of these patients.

\section{Study design}

This study is a prospective, multicentre, cohort study. Patients enrolled in the project will be followed up for 2 years. The depression and anxiety scales and the MACE will be collected at each follow-up visit. The study will be performed in 12 Class 3A (first-rate large general hospitals rated by the Ministry of Health of the People's Republic of China according to medical service and management, medical quality and safety, technical level and efficiency, and the scale of the hospital) comprehensive hospitals in the northwest region of China, which is led by the First Affiliated Hospital of Xi'an Jiaotong University. All participant centres have sufficient facilities, settings, and teams for the diagnosis and treatment of ACS and emotional disorders, and are capable of performing coronary angiography and PCI. The project has been approved by the ethics committees of the First Affiliated Hospital of Xi'an Jiao Tong University and registered at www.ClinicalTrials. gov.

\section{Patient and public involvement}

Patients were not involved in the design of this study and the development of the research questions and outcome measures nor will they be involved in the implementation of the study. The results will be disseminated to study participants by emails or letters, whichever they prefer.

\section{Box 1 Inclusion and exclusion criteria}

\section{Inclusion criteria}

1. Aged $>18$ years old.

2. Patients with a diagnosis of acute coronary syndrome including acute myocardial infarction (MI) and unstable angina (UA). Acute MI diagnosis must be met with at least two of these following criteria: typical chest pain (pain in the precordial area for more than $30 \mathrm{~min}$ ), elevated cardiac biomarkers (CK, CK-MB increases proportionally more than two times; troponin increases more than 10 times), the typical features of MI shown in ECG. The diagnosis of UA includes new onset angina within 2 months, crescendo angina, resting angina, infarction angina (angina occurring 24 hours to 1 month after $\mathrm{MI}$ ) and variant angina (angina pectoris caused by coronary spasm). Symptoms of angina pectoris are characterised by a progressive increase in the duration and intensity, but no myocardial enzyme elevation.

3. Good recovery after percutaneous coronary intervention (PCI): 1. stable vital sign after $\mathrm{PCl}$, including normal blood pressure, heart rate, temperature and respiratory rate; 2 . no angina pectoris onset; 3. no intervention complications.

4. Volunteer for the study and sign the informed consent.

\section{Exclusion criteria}

1. Severe heart failure, defined as left ventricular ejection fraction $\leq 30 \%$ or New York Heart Association class $\geq 11$.

2. Severe renal dysfunction, defined as creatinine clearance rate $\leq 30 \mathrm{~mL} / \mathrm{min}$.

3. Cancer (all kinds of malignant tumour).

4. Other severe mental illness including schizophrenia, severe dementia and substance abuse.

5. Bipolar disorder, depressive or anxiety disorders before PCI.

6. Ongoing administration of antipsychotic, antidepressant or antianxiety drugs.

7. Serious risk of suicide (the last item of Patient Health Questionnaire-9 scored over 2).

8. Severe, life-threatening medical condition (patients cannot participate in the study course).

9. Pregnancy and lactation.

\section{Participants}

Patients with a verified diagnosis of ACS within 7 days after PCI will be recruited. The recovery status of the recruited patients will be assessed up to 7 days after PCI before discharge in our study. The inclusion and exclusion criteria are listed in box 1 . The patients will be enrolled in the 12 collaborating hospitals from May 2017 to December 2019. The study flow chart is shown in figure 1. All participants will be asked to sign an informed consent form.

\section{Outcomes}

The primary outcome is the MACE results post-PCI, including all-cause mortality, MI, stroke, UA pectoris and unplanned revascularisation. The events will be evaluated at each follow-up, details of which include cause, onset, symptoms, treatments and the progress status, which will be verified with the corresponding medical records. If a patient suffered a primary outcome, then his or her study 


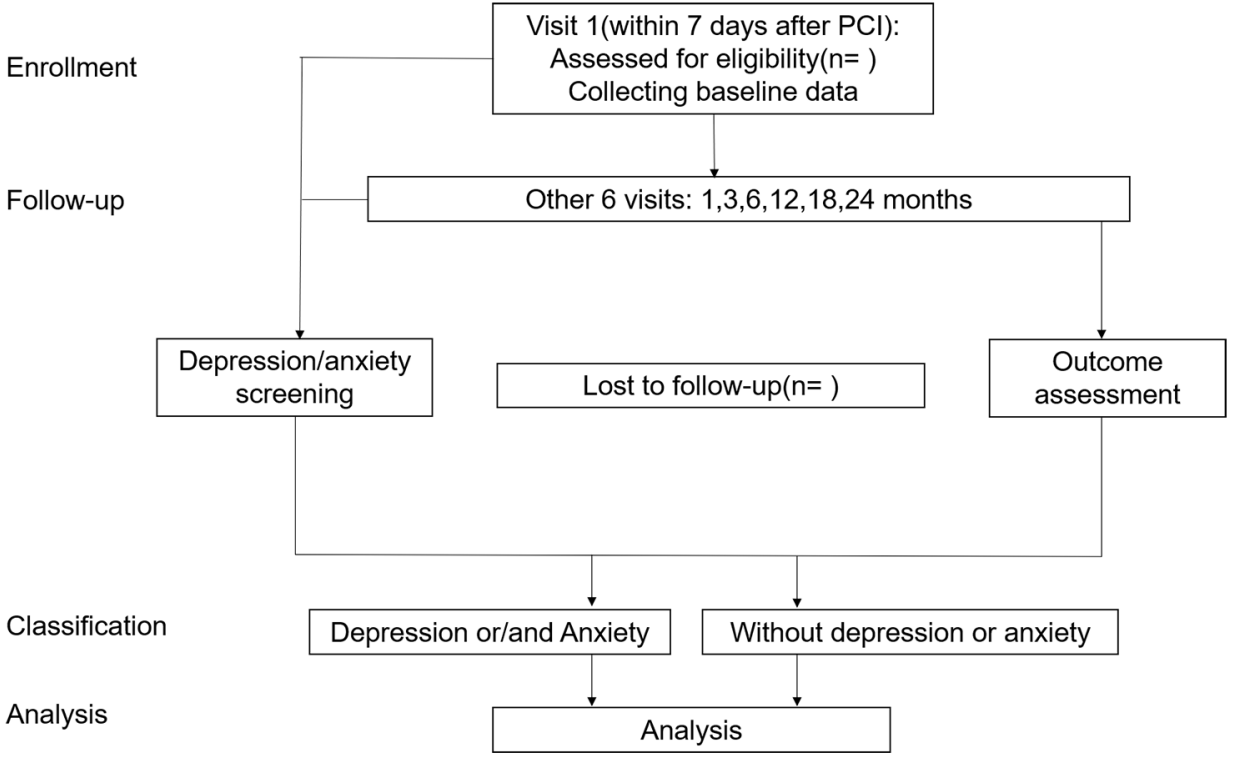

Figure 1 Study flow diagram.

process will be terminated on the day of the event after verification.

\section{Depression/anxiety measurements}

Anxiety and depression scales are assessed by the Generalised Anxiety Disorder (GAD-7) Assessment or GAD-2 and the Patient Health Questionnaire (PHQ-9) or PHQ-2, which will be obtained by self-rating at each visit.

\section{Self-rating anxiety scale}

The GAD-7 is composed of seven items according to the characteristics of GAD symptoms. Each item is scored as 0-3 according to the frequency of the respective issue from none to almost every day; thus, the total score of this scale ranges from 0 to 21. It is compact and reliable for identifying GAD and also has good validity when identifying other types of anxiety disorders. ${ }^{14}$ If a patient's GAD-7 score is over 9, which indicates moderate or severe anxiety for the patient, an appointment will be made with a psychiatrist for further treatment.

The GAD-2 scale includes the first two items of the GAD-7. The GAD-2 scale will be used at telephone follow-ups to evaluate anxiety with acceptable properties. $^{1415}$

\section{Self-rating depression scale}

The PHQ-9 was developed from the nine symptoms of depression according to the Diagnostic and Statistical Manual of Mental Disorders, 4th Edition (DSMIV) criteria. The scale is concise and operable to screen patients for depression and its severity with high reliability. ${ }^{16}$ Its scoring methods are the same as the GAD-7. If a patient's PHQ-9 score is over 9, which indicates moderate or severe depression for the patient, an appointment will be made with a psychiatrist for further treatment. Whenever the last item of the PHQ-9 of any patient scores is over 0 , which means a possible suicidal tendency, we will contact psychiatrists immediately to fully assess the patient's suicide risk, and appropriate intervention will be administered if necessary.

The PHQ-2 scale includes the first two items of the PHQ-9. The PHQ-2 scale will be used at telephone follow-ups to evaluate depression with acceptable properties. $^{1517}$

The GAD-7 and PHQ-9 will be self-rated in visit 1 in ward post-PCI, while the GAD-2 and PHQ-2 will be selfrated in the outpatient follow-up visit. If either the GAD-2 or PHQ-2 scores are over 2, such patients will be required to finish the whole PHQ-9 and GAD-7 scales.

\section{Other measurements}

Demographics (income level, occupation, educational level and marital status), medical history of mental illness and comorbidities and related family history will be collected at baseline only. Medication information on antidepressant or antianxiety medications, physical examination, laboratory examination and echocardiography will be collected at all visits (table 1). These variables will be used to adjust the statistical model and perform subgroup analyses.

\section{Follow-up}

Patients recruited in the study will receive standard ACS treatment, and be followed up at 1 month, 3 months, 6 months, 12 months, 18 months and 24 months after PCI. A telephone follow-up will be performed 1 week in advance before each outpatient visit to reduce the incidence of loss to follow-up. Additional visits to a psychiatry specialist will be scheduled for a patient if a patient's GAD-7 or PHQ-9 score is over 9. Further evaluation and treatment advices will be made by psychiatrists. Treatment of depression or anxiety is based on the patient's own will and is not mandatory. Subsequently, antidepressive or antianxiety treatment information will be recorded in 
Table 1 Evaluations at baseline and follow-up visits

\begin{tabular}{|c|c|c|c|c|c|c|c|}
\hline Time after $\mathrm{PCl}$ & 7 days & 1 month & 3 months & 6 months & 12 months & 18 months & 24 months \\
\hline Demographic information & $x$ & & & & & & \\
\hline $\begin{array}{l}\text { Medical history } \\
\text { (cardiac, mental illness, etc) }\end{array}$ & $x$ & & & & & & \\
\hline Family history & $x$ & & & & & & \\
\hline Comorbidities & $x$ & & & & & & \\
\hline $\begin{array}{l}\text { Laboratory examination } \\
\text { (AST, ALT, CRP, LDL-C, HDL, TC, } \\
\text { TG, FBG) }\end{array}$ & $x$ & $x$ & $x$ & $x$ & $x$ & $x$ & $x$ \\
\hline
\end{tabular}

ALT, alanine aminotransferase; AST, aspartate aminotransferase; CRP, high-sensitivity C reactive protein; FBG, fasting blood glucose; GAD, Generalised Anxiety Disorder; HDL-C, high-density lipoprotein cholesterol; LDL-C, low-density lipoprotein cholesterol; MI, myocardial infarction; PCl, percutaneous coronary intervention; PHQ, Patient Health Questionnaire; TC, total cholesterol; TG, triglycerides.

Case Report Forms (CRFs). All the patients will receive routine ACS treatment during the whole process.

\section{Data management}

Data will be collected locally in CRFs and entered into the central clinical centre by an electronic data capture system. The data will be monitored regularly for data quality control by a data management team at the centre. No additional data are collected if a participant discontinues this study or in other ways does not comply with the protocols.

\section{Sample size}

According to our preliminary research, about 20\% patients with ACS post-PCI developed depression and/ or anxiety $[\operatorname{Pr}(x=1)]$, the incidence of MACE in ACS patients without emotional disorders post-PCI was about $15 \%[\operatorname{Pr}(\mathrm{y}=1 \mid \mathrm{x}=0)]$. We presumed conservatively that the HR of MACE in 2 years for patients with depression and/or anxiety was 1.3 , and the rate of follow-up loss was approximately 20\%, a total of 5000 patients would yield power larger than $85 \%$ in a two-sided test at $\alpha$ of 0.05 .

\section{Statistical analysis}

All statistical tests will be two-tailed and significance will be considered as $p<0.05$. Continuous variables will be reported as the mean $\pm \mathrm{SD}$, and categorical variables will be reported as frequencies and percentages. Differences in demographic and clinical characteristics at baseline across groups will be tested using analysis of variance (ANOVA) for continuous variables and $\chi^{2}$ tests or Fisher exact tests for categorical variables.
Cox proportional hazard model with the diagnosis of depression and/or anxiety included as a time-dependent covariate will be used to determine if depression/anxiety post-PCI increases the risk of cardiovascular events among all patients. The time-dependent covariate indicates whether a patient has depression and/or anxiety at each time point. Patients without the two diseases will be the reference group. Within each group of patients with depression only, patients with anxiety only and patients with both diseases post-PCI, subgroup analyses will be performed to explore whether the freedom from MACE is different across different severity groups and whether the freedom from MACE is different between patients with and without treatment among patients with moderate and severe depression or anxiety. The Kaplan-Meier method will be used to determine the freedom from MACE. The repeated measure, mixed model of ANOVA will be used to determine if the PHQ-9 or GAD-7 changes over time.

\section{Ethics and dissemination}

In the case of important modifications to the protocol, trial investigators will be informed and the online registration will be updated. The results will be published in research articles or conference papers.

\section{DISCUSSION}

Studies have indicated that the occurrence of depression or anxiety after ACS is quite common. Post-ACS depression may increase the risk of cardiovascular events and mortality, while it is still inconclusive whether post-ACS 
anxiety has the same effect. Meanwhile, there is still a lack of evidence on whether the intervention towards post-ACS depression or anxiety could improve the cardiac prognosis of these patients. To our knowledge, this study is the first large-sample, multicentre, real-world cohort study on the impact of depression and/or anxiety in patients with ACS who have undergone PCI in China or in any developing country, and it will provide a basis for the diagnosis and treatment of these patients.

Anxiety and depression share similar risk factors and pathogenetic connections with ACS and increase cardiovascular risks of patients with ACS through both physiological and behavioural changes. ${ }^{8} 1819$ Depression and anxiety could lead to increased platelet activity, endothelial dysfunction, inflammation, reduced heart rate variability and neuroendocrine disorders, which may increase the risk of cardiovascular events. ${ }^{818}{ }^{19}$ Depression and anxiety could reduce the compliance of secondary prevention in patients with ACS, and patients are less likely to quit smoking, go on a diet, exercise, seek medical help and participate in cardiac rehabilitation. ${ }^{8}{ }^{19}$ Under the influence of these factors, the occurrence of depression or anxiety after PCI may lead to adverse cardiovascular events in patients with ACS. Therefore, antidepressant or antianxiety treatment might improve the prognosis of these patients.

Psychotherapy is of great significance in the treatment of depression and anxiety. However, it has little effect on the risk of cardiovascular events in post-ACS patients with anxiety or depression. ${ }^{20}{ }^{21}$ Selective 5-serotonin reuptake inhibitors (SSRIs), sertraline and citalopram could improve both depression symptoms and anxiety symptoms. Considering their safety and effects, in 2008, the AHA recommended sertraline and citalopram as firstline drugs in CHD patients with depression. ${ }^{12}$ SSRIs may have beneficial pleiotropic effects including recovery of heart rate variability, reduction of inflammatory markers, improvements in endothelial function and a reduction in platelet aggregation, which may provide cardiovascular protection in patients with PCI post-ACS. ${ }^{22}$ Nevertheless, it is still inconclusive whether SSRIs could have a beneficial effect on the cardiovascular prognosis in patients with post-ACS depression. ${ }^{23}{ }^{24}$ Recent studies have shown the risk of QT interval extension when using citalopram. ${ }^{25} \mathrm{In}$ addition, many adverse reactions to other kinds of antidepressants or antianxiety drugs could also have detrimental effects, which makes the recommended level less or even banned in post-ACS patients. ${ }^{82}$ Therefore, the administration of antidepressants needs prudent consideration. We intend to conduct subgroup analysis on the data of patients receiving antidepressive or antianxiety treatment according to their various therapies and their different responses after the end of the trial, so as to provide more sufficient evidence for the treatment of these patients.

In conclusion, this trial is the first large-sample multicentre real-world study in China or in any developing country, in which the occurrence of anxiety and depression in patients with PCI post-ACS will be recorded and the relationship between cardiovascular events and anxiety/ depression will be evaluated during 2 years of follow-up. Thus, our study is expected to provide solid and reliable data to support the clinical diagnosis and treatment of these patients.

\section{Author affiliations}

${ }^{1}$ Department of Cardiovascular Medicine, The First Affiliated Hospital of Xi'an Jiaotong University, Xi'an, China

${ }^{2}$ Key Laboratory of Environment and Genes Related to Diseases, Xi'an Jiaotong University, Ministry of Education, Xi'an, China

${ }^{3}$ Clinical Research Center, The First Affiliated Hospital of Xi'an Jiaotong University, Xi'an, China

${ }^{4}$ Key Laboratory of Molecular Cardiology, Xi'an Jiaotong University, Xi'an, China

Acknowledgements The authors thank all the participants and the patient advisors in this study.

Contributors The whole study design was led by ZY. The standard study process was made and checked by YX, JZ, JZ, YL and WL. XC and MG contributed to the follow-up part of the design. $X H$ and $Z Z$ contributed to the recruitment part of the design. This manuscript was written by YX and WL. All authors have reviewed the manuscripts, and the final version was approved by all.

Funding This study was supported by the Key Project of Research and Development Plan (2017ZDCXL-SF-02-04-01), Natural Science Foundation (No. 2018JM7063) of Shaanxi Province of China and the Clinical Research Award of the First Affiliated Hospital of Xi'an Jiaotong University, China (No. XJTU1AF-CRF-2016-004).

Competing interests None declared.

Patient consent for publication Not required.

Ethics approval This study is performed in agreement with the Helsinki Declaration (2000 edition). Before the start of the trial, the study protocol, informed consent and other research documents were examined and approved by the ethics committees of the First Affiliated Hospital of Xi'an Jiao Tong University which is responsible for this clinical trial under number XJTU1AF2016LSL-036, and also approved by the ethics committees of the other subcentre hospitals.

Provenance and peer review Not commissioned; externally peer reviewed.

Open access This is an open access article distributed in accordance with the Creative Commons Attribution Non Commercial (CC BY-NC 4.0) license, which permits others to distribute, remix, adapt, build upon this work non-commercially, and license their derivative works on different terms, provided the original work is properly cited, appropriate credit is given, any changes made indicated, and the use is non-commercial. See: http://creativecommons.org/licenses/by-nc/4.0/.

\section{REFERENCES}

1. Chen W, Gao R, Liu L, et al. Report for Chinese cardiovascular disease in 2014. Chin Circul J 2015;07:617-22.

2. Antoniou S, Colicchia M, Guttmann OP, et al. Risk scoring to guide antiplatelet therapy post-percutaneous coronary intervention for acute coronary syndrome results in improved clinical outcomes. Eur Heart J Qual Care Clin Outcomes 2018;4:283-9.

3. Zimmerman FH, Cameron A, Fisher LD, et al. Myocardial infarction in young adults: angiographic characterization, risk factors and prognosis (coronary artery surgery study registry). J Am Coll Cardiol 1995;26:654-61.

4. Meliga E, De Benedictis M, Gagnor A, et al. Long-term outcomes of percutaneous coronary interventions with stent implantation in patients $\leq 40$ years old. Am J Cardiol 2012;109:1717-21.

5. Lautamäki A, Airaksinen KEJ, Kiviniemi T, et al. Prognosis and disease progression in patients under 50 years old undergoing $\mathrm{PCl}$ : the CRAGS (coronary artery disease in young adults) study. Atherosclerosis 2014;235:483-7.

6. Liu M-yan, Jiang R-huan, Hu D-yi, et al. [Emotional disorder in patients with acute or stable coronary heart disease]. Zhonghua Xin Xue Guan Bing Za Zhi 2009;37:904-7.

7. Feng H-P, Chien W-C, Cheng W-T, et al. Risk of anxiety and depressive disorders in patients with myocardial infarction: a nationwide population-based cohort study. Medicine 2016;95:e4464. 
8. Huffman JC, Celano CM, Januzzi JL. The relationship between depression, anxiety, and cardiovascular outcomes in patients with acute coronary syndromes. Neuropsychiatr Dis Treat 2010;6:123-36.

9. Versteeg $\mathrm{H}$, Roest AM, Denollet J. Persistent and fluctuating anxiety levels in the 18 months following acute myocardial infarction: the role of personality. Gen Hosp Psychiatry 2015;37:1-6.

10. Jiang W, Krishnan RRK, O'Connor CM. Depression and heart disease: evidence of a link, and its therapeutic implications. CNS Drugs 2002;16:111-27.

11. van Melle JP, de Jonge P, Spijkerman TA, et al. Prognostic association of depression following myocardial infarction with mortality and cardiovascular events: a meta-analysis. Psychosom Med 2004;66:814-22.

12. Lichtman JH, Bigger JT, Blumenthal JA, et al. Depression and coronary heart disease: recommendations for screening, referral, and treatment: a science Advisory from the American heart association prevention Committee of the Council on cardiovascular nursing, Council on clinical cardiology, Council on epidemiology and prevention, and interdisciplinary Council on quality of care and outcomes research: endorsed by the American psychiatric association. Circulation 2008;118:1768-75.

13. Lichtman JH, Froelicher ES, Blumenthal JA, et al. Depression as a risk factor for poor prognosis among patients with acute coronary syndrome: systematic review and recommendations: a scientific statement from the American Heart Association. Circulation 2014;129:1350-69.

14. Plummer F, Manea L, Trepel D, et al. Screening for anxiety disorders with the GAD-7 and GAD-2: a systematic review and diagnostic metaanalysis. Gen Hosp Psychiatry 2016;39:24-31.

15. Kroenke K, Spitzer RL, Williams JBW, et al. An ultra-brief screening scale for anxiety and depression: the PHQ-4. Psychosomatics 2009;50:613-21.
16. Kroenke K, Spitzer RL, Williams JB. The PHQ-9: validity of a brief depression severity measure. J Gen Intern Med 2001;16:606-13.

17. Coulter SA, Campos K. Identify and treat depression for reduced cardiac risk and improved outcomes. Tex Heart Inst $J$ 2012;39:231-4.

18. Joynt KE, Whellan DJ, O'Connor CM. Depression and cardiovascular disease: mechanisms of interaction. Biol Psychiatry 2003;54:248-61.

19. Carney RM, Freedland KE. Depression and coronary heart disease. Nat Rev Cardiol 2017;14:145-55.

20. Berkman LF, Blumenthal J, Burg M, et al. Effects of treating depression and low perceived social support on clinical events after myocardial infarction: the enhancing recovery in coronary heart disease patients (ENRICHD) randomized trial. JAMA 2003;289:3106-16.

21. Lespérance F, Frasure-Smith N, Koszycki D, et al. Effects of citalopram and interpersonal psychotherapy on depression in patients with coronary artery disease: the Canadian cardiac randomized evaluation of antidepressant and psychotherapy efficacy (CREATE) trial. JAMA 2007;297:367-79.

22. Teply RM, Packard KA, White ND, et al. Treatment of depression in patients with concomitant cardiac disease. Prog Cardiovasc Dis 2016:58:514-28.

23. Taylor CB, Youngblood ME, Catellier D, et al. Effects of antidepressant medication on morbidity and mortality in depressed patients after myocardial infarction. Arch Gen Psychiatry 2005;62:792-8.

24. Glassman $\mathrm{AH}, \mathrm{O}^{\prime}$ Connor $\mathrm{CM}$, Califf RM, et al. Sertraline treatment of major depression in patients with acute MI or unstable angina. JAMA 2002;288:701-9.

25. Cooke MJ, Waring WS. Citalopram and cardiac toxicity. Eur J Clin Pharmacol 2013;69:755-60. 